\title{
Numerical investigations on the vortex-induced vibration of moving square cylinder by using incompressible lattice Boltzmann method
}

\author{
Xiaohai Jiang ${ }^{\mathrm{a}, \mathrm{b}}$, Yiannis Andreopoulos ${ }^{\mathrm{a}, *}$, Taehun Lee ${ }^{\mathrm{a}}$, Zhexuan Wang ${ }^{\mathrm{a}}$ \\ ${ }^{a}$ Department of Mechanical Engineering, City College of City University of New York, New \\ York, 10031, USA \\ ${ }^{b}$ Key Laboratory of Transient Physics, Nanjing University of Science and \\ Technology,Nanjing,210094,China
}

\begin{abstract}
Vortex-induced vibrations (VIV) phenomena related to self-excited energy harvesters consisting of square cylinders have been investigated numerically by using the BGK incompressible lattice Boltzmann method. In the present work, such a harvester is placed inside a channel flow and is allowed to oscillate without a structural restoring force in a direction normal to the flow. Currently the half-way bounce-back boundary and interpolations method are being used to model the moving boundary. The numerical results of the periodical and nonperiodical oscillations and the frequency content of the longitudinal and lateral forces acting on the square cross section harvester are discussed in detail. The numerical technique was validated by computing the flow around a fixed cylinder. The results are compared favorably with the results obtained by classical CFD methods.
\end{abstract}

Keywords: square cylinder, vortex-induced vibrations, lattice Boltzmann

\footnotetext{
${ }^{*}$ Corresponding author

Email address: andre@ccny.cuny.edu (Yiannis Andreopoulos)
}

Preprint submitted to Journal of Computers \& Fluids 
method

\section{Introduction}

Vortex-induced vibration (VIV) is a self-sustained vibration, generally appeared in nature and in phenomena related to flag fluttering, cable swing, and tree waving in the wind. Understanding the physical mechanisms and interactions in VIV is important to several industries, such as offshore structures, bridges, submarines, heat exchangers and other applications. Recently, VIV is used in a family of energy harvesters consisting of a cantilever beam with a bluff body at its tip [1, 2, 3. In energy harvesting, the self-sustained vibration of the oscillator is expected to be periodic for a stable energy transfer.

There are many published works in the literatures on experimental and numerical investigations of the VIV phenomena 4, 5, 6, 7, 8, 9, 10, 11. Due to the complex, non-linear interactions between the structural motions and the vortex-shedding [4, it is very difficult to predict the vortex-induced vibration in modeling the flow field, structural vibration and fluid-structure interaction [12. In most of previous works the traditional CFD numerical methods are used to solve the VIV problems. Bishop and Hassan[13] studied the effect of the transverse force to the oscillation of the circular cylinder and found that a jump phase occurs when the oscillation frequency approached the shedding frequency. The so called "lock-in" phenomenon is traditionally referred to the resonance between the flow and structure 9 , i.e. the frequency superposition of the Strouhal shedding and structure vibration, and it is generally associated 
with VIV. In addition, there always exists a frequency doubling relationship between cross-flow and in-line response under lock-in conditions [4]. Meneghini and Bearman[14] adopted a discrete vortex method incorporating viscous diffusion to 25 simulate the flow about an oscillating circular cylinder and an asymmetric mode of shedding was observed. Vortex shedding from a transversely oscillating circular cylinder in a uniform flow has been studied by solving the two-dimensional unsteady Navier-Stokes equations with a primitive-variable formulation by $\mathrm{Lu}$ and Dalton[15].

Similar to above mentioned circular cylinder cases, the VIV involving square cylinder was also studied by experiments [6, 7, 16] and numerical simulations [10, 11. The effects of axial applied tension on the vibration properties during vortex-induced vibration of a horizontally mounted square cylinder was studied experimentally in the Reynolds number range $(\operatorname{Re}=1000 \sim 16000)[6$. Experiments focusing on the effects of the angle of attack, relatively to the incoming flow of a square cylinder on the cylinder's flow-induced vibration were performed by András Nemes et al.[7]. Alam et al.[16] performed a comprehensive experimental study of the wake of two side-by-side square cylinders. The wake of the fixed square cylinder was simulated numerically by using a standard centered and second-order-accurate finite-difference scheme on a staggered Cartesian mesh to solve the incompressible, two-dimensional N-S equations [10. In this study, the inversion phenomenon of the vortex street and its position characteristics were discussed in detail. Generally, the cylinder oscillation is due to the alternative change of the sign of forces (i.e. lift force) 

due to the vortex shedding in the wake, which is dominated by the incoming flow velocity or Reynolds number. That is to say, self-sustained oscillation is related directly to the vortex shedding. Sánchez-sanz and Velazquez [1] used the mixed implicit-explicit relaxation-based pseudo-compressibility formulation to simulate the vortex-induced oscillation of a square section cylinder placed inside a two-dimensional channel flow. The oscillation modes (periodic and non-periodic) related to the mass/density ratios of the cylinder and fluid were discussed in details under a Reynolds range $\operatorname{Re}=50 \sim 200$. A detailed review of the traditional numerical simulations about the flow past a cylinder associated with VIV can be found in references [5, 8, 17, 18. ditional CFD with some advantages. There are few numerical investigations on VIV by using LBM[19, 20, 21]. Breuer et al.[19] investigated the laminar flow past a fixed square cylinder by using lattice Boltzmann and finite-volume method under the Reynold number less than 300. Islam et al. [20] used incom60 pressible lattice Boltzmann method to studied numerically a uniform flow past a fixed rectangular cylinder with different aspect ratios. Flow across a row of transversely oscillating square cylinders was simulated numerically by using the lattice Boltzmann method with a peculiar process, which means that the flow is oscillating with respect to the cylinders by avoiding the actual movement of 65 the cylinders with respect to the computational grid 21].

In the present work, VIV phenomena of the flow around a rigid-body cylinder (oscillator) with square section moving in cross-flow direction in the channel 
flow were simulated numerically by using BGK incompressible lattice Boltzman$\mathrm{n}$ method. The half-way bounce-back boundary and interpolations method were used to process the square cylinder motion relative to the computational grid. In particular, we have investigated the flow around a square cylinder undergoing forced oscillations in a Poiseuille flow. Three different cases were considered in our investigations. First, the case of a stationary square cylinder in a Poiseuille flow was investigated with the objective to validate the numerical method; second the case of a moving square cylinder in a quiescent flow was computed to establish the self-induced vortex interaction by the motion of the cylinder in the absence of cross Poiseuille flow. Last the case of a moving square cylinder inside a Poiseuille flow was investigated and compared with existing results.

\section{Numerical methods}

The lattice Boltzmann equation with Bhatnagar-Gross-Krook(BGK) single relaxation time (SRT) is used in current research

$$
\begin{gathered}
f_{i}\left(t+\delta_{t}, \mathbf{x}+\mathbf{e}_{i} \delta_{t}\right)=f_{i}(t, \mathbf{x})+\frac{1}{\tau}\left(f_{i}^{e q}(t, \mathbf{x})-f_{i}(t, \mathbf{x})\right), i=0,1,2 \ldots 8 \\
f_{i}^{e q}(f)=H_{i}^{e q}(\rho(f), \mathbf{u}(f))
\end{gathered}
$$

Here, $f_{i}(t, \mathbf{x})$ is the mesoscopic variable, indicating the probability density distribution of $i$-component of the discretization velocity space at time $t$ and position $\mathbf{x}$. The discrete time-step $\delta_{t}$ is set equal to unity as well as the lattice spacing $\delta_{x}$ 
$\left(\delta_{t}=\delta_{x}=1\right)$. The equilibrium values, $H_{i}^{e q}$, are obtained through the following

$$
H_{i}^{e q}(\rho, \mathbf{u})=w_{i} \rho\left(1+c_{s}^{-2} \mathbf{e}_{i} \cdot \mathbf{u}+\frac{c_{s}^{-4}}{2}\left(\left|\mathbf{e}_{i} \cdot \mathbf{u}\right|^{2}-c_{s}^{2} \mathbf{u}^{2}\right)\right)
$$

Where, $\mathbf{u}$ and $\rho$ is the fluid velocity and density. $\mathbf{e}_{i}$ is the velocity component in the lattice velocity space, which is dependent on the velocity discretization 90 model, for D2Q9 model (as shown in Fig. 1)

$$
\mathbf{e}_{i}= \begin{cases}0 & \text { for } i=0 \\ c(\cos ((i-1) \pi / 4), \sin ((i-1) \pi / 4)) & \text { for } i=1 \sim 4 \\ \sqrt{2} c(\cos ((i-1) \pi / 4), \sin ((i-1) \pi / 4)) & \text { otherwise. }\end{cases}
$$

Accordingly weighted parameters $w_{i}$ are given as

$$
w_{i}= \begin{cases}4 / 9 & \text { for } i=0 \\ 1 / 9 & \text { for } i=1-4 \\ 1 / 36 & \text { otherwise }\end{cases}
$$

The macroscopic variables, i.e., the fluid density and momentum are

$$
\rho=\Sigma_{i} f_{i}=\Sigma_{i} f_{i}^{e q}, \quad \rho \mathbf{u}=\Sigma_{i} \mathbf{e}_{i} f_{i}=\Sigma_{i} \mathbf{e}_{i} f_{i}^{e q}
$$

And the relationship of the viscous and relaxation time is

$$
\nu=(2 / \zeta-1) \delta_{x} \cdot c / 6
$$

Here, $\zeta \equiv \delta_{t} / \tau, c=\delta_{x} / \delta_{t}=1$. The pressure was obtained through the equation

of state: $p=c_{s}^{2} \rho$, here $c_{s}$ is the sound speed, in D2Q9 model, $c_{s}=c / \sqrt{3}$. That is to say the density is related to the pressure even if the flow is incompressible, i.e., the compressibility effects are embedded in LBM. To reduce or to eliminate 
the compressible effect, we use the He-luo model[22], in which the equilibrium values are calculated through

$$
H_{i}^{e q}(\rho, \mathbf{u})=w_{i}\left(\rho+\rho_{0}\left(c_{s}^{-2} \mathbf{e}_{i} \cdot \mathbf{u}+\frac{c_{s}^{-4}}{2}\left(\left|\mathbf{e}_{i} \cdot \mathbf{u}\right|^{2}-c_{s}^{2} \mathbf{u}^{2}\right)\right)\right)
$$

100 velocity is $\rho_{0} \mathbf{u}=\Sigma_{i} \mathbf{e}_{i} f_{i}$. An additional condition must be satisfied [22],

$$
|\mathbf{u}| \ll c_{s}, T \gg L / c_{s}
$$

Where, $T$ and $L$ is time and length scale of the macroscopic change, respectively.

\subsection{Boundary conditions}

The computational domain and boundary conditions are shown as in Fig 2 The left boundary is velocity specified inflow boundary condition 23$]$ with Poiseuille flow profile, and the right outflow boundary is zero-extrapolation boundary, the top and bottom boundaries are solid boundary by using the on-site bounceback boundary method. The moving boundaries of the cylinder are processed by using the interpolations method recommended by 24$]$. For the velocity specified boundary (in Fig. 3a), the relationship between the populations and macrovariabls are there,

(1) $\quad \rho=f_{0}+f_{\mathbf{1}}+f_{2}+f_{3}+f_{4}+f_{\mathbf{5}}+f_{6}+f_{7}+f_{\mathbf{8}}$

(2) $\quad \rho u=f_{1}-f_{3}+f_{5}-f_{6}-f_{7}+f_{8}$

(3) $\rho v=f_{2}-f_{4}+f_{5}+f_{6}-f_{7}-f_{8}$

(4) $u=u_{0}(y)$

(5) $\quad v=0$

(6) $f_{1}-f_{1}^{e q}=f_{3}-f_{3}^{e q} \quad\left(\right.$ Bounceback : $\left.f_{1}^{n e q}=f_{3}^{n e q}\right)$ 
Based on the above equations, the unsolved populations and pressure (density) can be obtained from

$$
\left\{\begin{array}{l}
f_{1}=f_{3}+\frac{2}{3} \rho u_{0}(y) \\
f_{5}=f_{7}-\frac{1}{2}\left(f_{2}-f_{4}\right)+\frac{1}{6} \rho u_{0}(y)+\frac{1}{2} \rho v \\
f_{8}=f_{6}+\frac{1}{2}\left(f_{2}-f_{4}\right)+\frac{1}{6} \rho u_{0}(y)-\frac{1}{2} \rho v \\
\rho=\left[f_{0}+f_{2}+f_{4}+2\left(f_{3}+f_{6}+f_{7}\right)\right] /\left(1-u_{0}(y)\right) \quad \text { or } \\
\rho=\left[f_{0}+f_{2}+f_{4}+2\left(f_{3}+f_{6}+f_{7}\right)\right]+\rho_{0} \quad(\text { Incompressible LBM })
\end{array}\right.
$$

Particular attention should be given to the corners (Fig. 3b), as Point A

$$
\begin{cases}\text { (1) } & \rho=f_{0}+f_{1}+f_{\mathbf{2}}+f_{\mathbf{3}}+f_{4}+f_{5}+f_{\mathbf{6}}+f_{7}+f_{\mathbf{8}} \\ \text { (2) } & \rho u=f_{1}-f_{3}+f_{5}-f_{6}-f_{7}+f_{8} \\ \text { (3) } & \rho v=f_{2}-f_{4}+f_{5}+f_{6}-f_{7}-f_{8} \\ \text { (4) } & u=0 \\ \text { (5) } & v=0 \\ \text { (6) } & f_{1}=f_{3}, f_{4}=f_{2}, f_{8}=f_{6} \quad \text { (Bounceback) }\end{cases}
$$

115 Then the unsolved populations are

$$
f_{7}=f_{5}=\frac{1}{2}\left[\rho-f_{0}-2\left(f_{2}+f_{3}+f_{6}\right)\right]
$$

Similarity operations on point B, then

$$
f_{8}=f_{6}=\frac{1}{2}\left[\rho-f_{0}-2\left(f_{3}+f_{4}+f_{7}\right)\right]
$$

Same procedures should be also applied to the corners of the outflow boundary as well as the inflow boundary.

For the moving solid boundary of the cylinder, we use the second-order 120 interpolations method [24]: 


$$
\begin{cases}q<\frac{1}{2}, \quad & f_{\bar{i}}\left(t, \mathbf{x}_{j}\right)=q(1+2 q) f_{i}\left(t, \mathbf{x}_{j}+\mathbf{e}_{i} \delta t\right)+\left(1-4 q^{2}\right) f_{i}\left(t, \mathbf{x}_{j}\right) \\ & -q(1-2 q) f_{i}\left(t, \mathbf{x}_{j}-\mathbf{e}_{i} \delta t\right)+3 w_{a} \rho_{0}\left(\mathbf{e}_{\bar{i}} \cdot \mathbf{u}_{w}\right) \\ q \geq \frac{1}{2}, \quad & f_{\bar{i}}\left(t, \mathbf{x}_{j}\right)=\frac{1}{q(2 q+1)} f_{i}\left(t, \mathbf{x}_{j}+\mathbf{e}_{i} \delta t\right)+\frac{2 q-1}{q} f_{\bar{i}}\left(t, \mathbf{x}_{j}-\mathbf{e}_{i} \delta t\right) \\ & -\frac{2 q-1}{2 q+1} f_{\bar{i}}\left(t, \mathbf{x}_{j}-2 \mathbf{c}_{i} \delta t\right)+\frac{3 w_{i} \rho_{0}}{q(2 q+1)}\left(\mathbf{e}_{\bar{i}} \cdot \mathbf{u}_{w}\right) \\ q \equiv \quad & \frac{\left|\mathbf{x}_{j}-\mathbf{x}_{w}\right|}{\delta_{x}}, q \in(0,1]\end{cases}
$$

Here, $\mathbf{x}_{j}$ is the position of the boundary node[25] while $\mathbf{x}_{w}$ is the wall position. $f_{\bar{i}}$ denotes the distribution functions of the velocity $\mathbf{e}_{\bar{i}} \equiv-\mathbf{e}_{i} \cdot \mathbf{u}_{w}$ is the velocity of the moving wall.

Another issue which needs to be addressed is how to determine and differentiate the populations of the nodes from the solid nodes (in boundary) to fluid ones (as shown in Fig 4). The populations of the nodes includes equilibrium and non-equilibrium parts, i.e., $f_{i}^{e q}, f_{i}^{n e q}$. The former is calculated from the macrovariables (density,velocity ) through linear interpolations from its neighbors, and the latter is also from interpolations of the non-equilibrium parts

${ }_{130} f_{i}^{n e q}=f_{i}-f_{i}^{e q}$. Then the unsolved populations can be obtained from the sum of both.

\subsection{Calculation of the forces on the rigid body}

In the present work we assume that the motion of the cylinder is in the y-direction only and is governed by the Newton's second law,

$$
\left\{\begin{array}{l}
M_{s} a_{y}=F_{y} \\
a_{y}=\frac{d^{2} Y_{c}}{d t^{2}}=F_{y} / M_{s}=F_{y} /\left(m \rho_{\infty} V_{s}\right)
\end{array}\right.
$$


Here, $Y_{c}, a_{y}$ are the displacement and acceleration of the cylinder's centroid in y-direction. $M$ and $V_{s}$ are the mass and volume of the cylinder. $m$ denotes density ratio between the cylinder and flow density, i.e. $m=\frac{\rho_{s}}{\rho_{\infty}} \cdot F_{y}$ is ycomponent of the force $(F)$ on the cylinder body, which has been calculated by using two methods, the traditional method[26] from the macro variables and the Momentum-Exchange Algorithm (MEA) method 25, 27, 28, from the populations directly. In the first case with the traditional method [26], the force is computed as

$$
\left\{\begin{aligned}
\mathbf{F}_{S} & =\int_{\Gamma(t)}(-p(t, x) \mathbf{I})+\mathbf{S}(t, \mathbf{x}) \cdot \mathbf{n}(x) d \sigma(\mathbf{x}) \\
\mathbf{S} & =\nu\left(\nabla \mathbf{u}+\nabla \mathbf{u}^{T}\right)
\end{aligned}\right.
$$

While in the Momentum-Exchange Algorithm[25] (Fig. 5) is

$$
\left\{\begin{aligned}
\phi_{i}\left(t, \mathbf{x}_{j}\right) & =\mathbf{e}_{i} f_{i}^{c}\left(t, \mathbf{x}_{j}\right)-\mathbf{e}_{\bar{i}} f_{\bar{i}}\left(t+\delta_{t}, \mathbf{x}_{j}\right) \\
& =\mathbf{e}_{i}\left(f_{\bar{i}}\left(t+\delta_{t}, \mathbf{x}_{j}\right)\right)+f_{i}^{c}\left(t, \mathbf{x}_{j}\right) \\
\mathbf{F} & =\sum_{\left(\mathbf{x}_{j}, i\right) \in B(\Gamma)} \phi_{i}\left(t, \mathbf{x}_{j}\right) .
\end{aligned}\right.
$$

\section{Results and Discussion}

\subsection{A fixed square cylinder in a Poiseuille flow}

To validation the numerical method above, the cases of a Poiseuille flow over the fixed cylinder are simulated numerically in different Reynolds numbers $(\mathrm{Re}=70 \sim 166)$. The computational domain and boundary conditions are shown in the same as the the following moving cylinder in a Poiseuille flow (as shown in Fig 2 with blockage ratio $\beta=1 / 8$. The velocity of the inflow (left boundary) is specified by fully developed Poiseuille profile with the maximum speed $u_{c}=$ 
0.1 at the center. And the initial populations are initialized by equilibrium values of the zero velocity. When the lift force of the square cylinder become periodic(i.e. Fig 6 ), the calculation will be terminated. In Fig 6, the lift histories 155 were calculated by traditional method and Momentum-Exchange Algorithm. The result of MEA is consistent with the one of traditional method and their difference is no more than $2.0 \%$. In the following, the MEA was adopted to compute the force of the square cylinder.

The Strouhal number was calculated as $S t=\frac{f D}{u_{c}}$, where $D$ and $u_{c}$ are the diameter of the cylinder and the maximum speed of the inflow profile respectively. $f$ is the vortex shedding frequency which is obtained by using Fast Fourier Transform (FFT) of the lift force acting on the cylinder.

Fig]7 is the Strouhal number at different Reynolds number under different grid number. Here, $\eta$ denotes the ratio of grid number which compares to the grid $960 \times 160$. The curves of Strouhal number at $\eta=1,9 / 4,4$ are also consistent $(\operatorname{Re}=70 \sim 166)$. And in $\eta=9 / 4$ and 4 , their difference is very small and no more than $0.6 \%$. So the finer grid number $1920 \times 320(\eta=4)$ is adopted. Fig 8 shows the calculated results by using the current LBM. These results are also compared to results obtained by using traditional CFD methods 10, 19. The trend of the $S t$ changes with increasing the Reynolds number in current LBM method is consistent with the tractional CFD results. A good agreement was obtained with no more than $2.9 \%(\operatorname{Re}=70 \sim 166)$ difference to the results of traditional CFD methods by [10]. 


\subsection{A moving square cylinder in a quiescent background flow}

175

velocity relation by integration. It appears that the numerical displacement of the cylinder is in good agreement with the analytical results, and their difference is no more than $3.1 \%$. 
After the two test validation cases were completed, the code was used to compute the vortical flow structure when the cylinder was free to vibration in the y-direction by the VIV caused by the cross channel flow in the $\mathrm{x}$-direction.

In this case, the distance of the cylinder to the inflow boundary was $l=7.5 h$,

as shown in Fig2 Its height and length of the channel flow are $H=2.5 h$ ( $\beta=2 / 5), L=22.5 h$ respectively. In this work the height of the cubic cylinder is $h=40$. The boundary and initial conditions are the same as the aforementioned cases of a fixed square cylinder in a Poiseuille flow.

Fig 13 is the curve of Strouhal number under different grid number $(\eta=1$ denotes the case $900 \times 100)$ at $\operatorname{Re}=200$ before the square cylinder moving. It is shown that the value of the Strouhal number tends to be a constant (0.432) when $\eta$ is more than 0.64 . And this constant is very close to the value (0.412) in [11. So the lattice number $900 \times 100(\eta=1)$ is applied to the following cases.

There exist two modes of oscillation along the transversal directions of the flow about the cylinder motion induced by vortex. One is the periodical oscillations and the other one is non-periodical, depending on the mass of the cylinder and flow characteristics. In the following, we will discuss these oscillations.

In the periodical oscillations, the Reynolds number and ratio of the cylinder to the fluid density were $\operatorname{Re}=200, m=0.7$, respectively. In [11, if the density ratio is $m \geq m_{c}\left(m_{c}=0.31\right)$, the cylinder motion will follow a periodical pattern under $\operatorname{Re}=200$. Fig 14 shows the history of the cylinder's centroid displacement $\left(Y_{c}\right)$, and Fig 15 shows its FFT. The non-dimensional frequency number (i.e. 
Strouhal number) of the cylinder motion is $f_{y_{m}}^{*}=0.4500$.

In order to show the relationship between the frequency of cylinder's motion and its natural frequency (vortex shedding frequency), the case with the cylinder fixed on the centerline of the channel flow was computed. Fig, 16 shows the history of the lift force $\left(F_{y}\right)$ and drag force $\left(F_{x}\right)$ on the fixed cylinder. Their motions are periodic with different frequencies and phase difference. The corresponding FFT of $F_{x}$ and $F_{y}$ are shown in the Fig 17 and Fig 18 , respectively. In both cases the non-dimensional oscillation frequencies are $f_{x_{s}}^{*}=0.8639, f_{y_{s}}^{*}=0.4320$, respectively. Furthermore, the vortex shedding frequency (i.e. natural frequency) of the vortex street of the wake flow is $f_{v o r}^{*}=f_{y_{s}}^{*}=0.4320$. Compared to the motion frequency of the aforementioned moving cylinder, they are almost equal, $f_{y m}^{*} \approx f_{v o r}^{*}$. The self-sustained oscillation of the moving cylinder in $\operatorname{Re}=200$ is in the so-called Lock-in mode [9] and the frequency of the longitudinal force appears to be twice the frequency of the lateral force as symmetry requires [4, i.e., $f_{x_{s}}^{*} \approx 2 f_{y_{s}}^{*}$.

Different oscillations with somehow irregular motions appear when the density ratio is less than critical density ratio $\left(m_{c}=0.31\right.$ in $\left.R e=200[11]\right)$. Fig 19 shows the history of the cylinder's centroid position in $\mathrm{Re}=200, m=0.05$. Its FFT is shown in Fig 20. The displacement of the cylinder centroid is not regular, and there exist several frequency peaks in Fig 20. That is to say, the irregular motion is compose of several resonance oscillations. 


\section{Conclusion}

Boltzmann method used in present work is capable to simulate the vortexinduced vibration phenomena. In particular the following conclusions can be drawn.

(1) For the fixed cylinder cases in the range of $\operatorname{Re} \leq 166$, the calculated result$\mathrm{s}$ are in good agreement with results of traditional CFD methods[10, 21]. When the Reynolds number is Re> 166, the trend of the Strouhal number variation with Reynolds number is still consistent with the traditional results, but their differences increases with Reynolds number. Perhaps it is related to the size of computational domain or the inlet/outlet boundary conditions.

(2) For the cases with the lateral movement cylinder, there also exist two types of self-sustained oscillations as found in the results using traditional CFD methods [11, i.e., periodical and non-periodical ones, which depend on the density ratios of the cylinder to that of the fluid. The former oscillations appear in the case of relatively large density ratios, while the latter appears in the case of small density ratios.

(3) For the case of the lateral periodical oscillation, the dimensionless vibration frequency is approximately equal to the Strouhal number of fixed cylinder under the same computational conditions. In this case, the frequency of longitudinal force (drag) is twice the frequency of the lateral force (lift)

From the above numerically investigated cases, it can be seen that the lattice which is typical of lock-in phenomena, where the flow field is in a resonance 
state and the amplitude of the oscillation reaches its maximum.

\section{Acknowledgement}

The work was sponsored by the National Science Foundation (CBET \#1033117) and a fellowship support from China Scholarship Council.

\section{References}

[1] D. Akaydin, N. Elvin, Y.Andreopoulos, Wake of a cylinder: A paradigm for energy harvesting with piezoelectric materials, Experiments in Fluids 49 (1) (2010) 291-304.

[2] D. Akaydin, N. Elvin, Y.Andreopoulos, Performance of a self-excited fluidic energy harvester, Journal of Smart Materials and Structures 21 (2) (2012) 25007-25019. doi:10.1088/0964-1726/21/2/025007.

[3] H. D. Akaydın, N. Elvin, Y. Andreopoulos, Flow-induced vibrations for piezoelectric energy harvesting, in: Advances in Energy Harvesting Methods, Springer, 2013, pp. 241-267.

[4] J. Vandiver, J.-Y. Jong, The relationship between in-line and cross-flow vortex-induced vibration of cylinders, Journal of Fluids and Structures 1 (1987) 381-399.

[5] C. Williamson, R. Govardhan, A brief review of recent results in vortexinduced vibrations, Journal of Wind Engineering and Industrial Aerodynamics 96 (2008) 713-735. doi:10.1016/j.jweia.2007.06.019. 
[6] B. Sanaati, N. Kato, Vortex-induced vibration(viv) dynamics of a tensioned flexible cylinder subjected to uniform cross-flow, Journal of Marine Science and Technology 18 (2013) 247-261. doi:10.1007/s00773-012-0204-z. jfm.2012.353

[8] C. Williamson, R. Govardhan, Vortex-induced vibrations, Annu. Rev. Fluid flow-induced vibration mechanisms of a square cylinder with varying angles of attack, Journal of Fluid Mechanics 710 (2012) 102-130. doi:10.1017/ Mech. 36 (2004) 413-455.

[9] M. R. Gharib, Vortex-induced vibration, absence of lock-in and fluid force deduction, Ph.D. thesis, California Institute of Technology (1999).

[10] S. Camarri, F. Giannetti, On the inversion of the von kármán street in the wake of a confined square cylinder, Journal of Fluid Mechanics 574 (2007) 169-178. doi:10.1017/S0022112006004058

[11] M. Sánchez-sanz, A. Velazquez, Vortex-induced vibration of a prism in internal flow, Journal of Fluid Mechanics 641 (2009) 431-440. doi:10. $1017 / \mathrm{s} 0022112009991893$.

[12] R. So, X. Wang, Vortex-induced vibrations of two side-by-side eulerbernoulli beams, Journal of Sound and Vibration 259 (2003) 677-700. doi:10.1006/jsvi.2002.5099.

[13] R. Bishop, A. hassan, The lift and drag forces on a circular cylinder oscil- 
lating in a flowing fluid, Proceedings of the Royal Society of London Series A 277 (1964) 51-75.

305

[14] J.R.Meneghini, P.W.Bearman, Numerical simulation of high amplitude oscillatory flow about a circular cylinder, Journal of fluids and structures 9 (1995) 435-455.

[15] X. Lu, C. Dalton, Calculation of the timing of vortex formation from an oscillating cylinder, Journal of Fluids and Structures 10 (1996) 527-54.

[16] M. Alam, Y. Zhou, X. Wang, The wake of two side-by-side square cylin-

1. ders, Journal of Fluid Mechanics 669 (2011) 432-471. doi:10.1017/ S0022112010005288.

[17] R. Gabbai, H. Benaroya, An overview of modeling and experiments of vortex-induced vibration of circular cylinders, Journal of Sound and Vibration $282(2005)$ 575-616. doi:10.1016/j.jsv.2004.04.017.

[18] S. Kim, P. Wilson, Z.-M. Chen, Numerical simulation of force and wake mode of an oscillating cylinder, Journal of Fluids and Structures 44 (2014) 216-225. doi:10.1016/j.jfluidstructs.2013.11.010.

[19] M. Breuer, J. Bernsdorf, T. Zeiser, F. Durst, Accurate computations of the laminar flow past a square cylinder based on two different methods: latticeboltzmann and finite-volume, International Journal of Heat and Fluid Flow 21 (2000) 186-196. doi:10.1016/S0142-727X (99) 00081-8.

[20] S. U. Islam, C. Zhou, A. Shah, P. Xie, Numerical simulation of flow past 
rectangular cylinders with different aspect ratios using the incompressible lattice boltzmann method, Journal of mechanical science and technology 26 (4) (2012) 1027-1041.

[21] C. Sewatkar, A. Sharma, A. Agrawal, Simulation of flow across a row of transversely oscillating square cylinders, Journal of Fluid Mechanics 680 (2011) 361-397. doi:10.1017/jfm.2011.167.

[22] X. He, L.-S. Luo, Lattice boltzmann model for the incompressible navierstokes equation, Journal of Statistical Physics 88 (1997) 927-944.

[23] Q. Zou, X. He, On pressure and velocity boundary conditions for the lattice boltzmann bgk model, Physics of Fluids 9 (6) (1997) 1591-1598.

[24] P. Lallemand, L.-S. Luo, Lattice boltzmann method for moving boundaries, Journal of Computational Physics 184 (2003) 406-421.

[25] A. Caiazzo, M. Junk, Boundary forces in lattice boltzmann: Analysis of momentum exchange algorithm, Computers \& Mathematics with Applications 55 (2008) 1415-1423. doi:10.1016/j.camwa.2007.08.004.

[26] T. Inamuro, K. Maeba, F. Ogino, Flow between parallel walls containing the lines of neutrally buoyant circular cylinders, International Journal of Multiphase Flow 26 (2000) 1981-2004.

[27] A. Ladd, Numerical simulations of particulate suspensions via a discretized boltzmann equation. part 1. theoretical foundation, Journal of Fluid Mechanics 271 (1994) 285-309. doi:10.1017/S0022112094001771. 


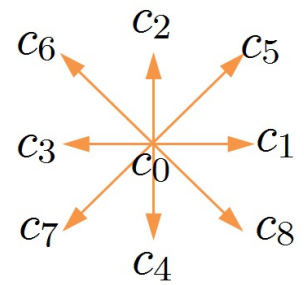

Figure 1: Velocity components of the D2Q9 model.

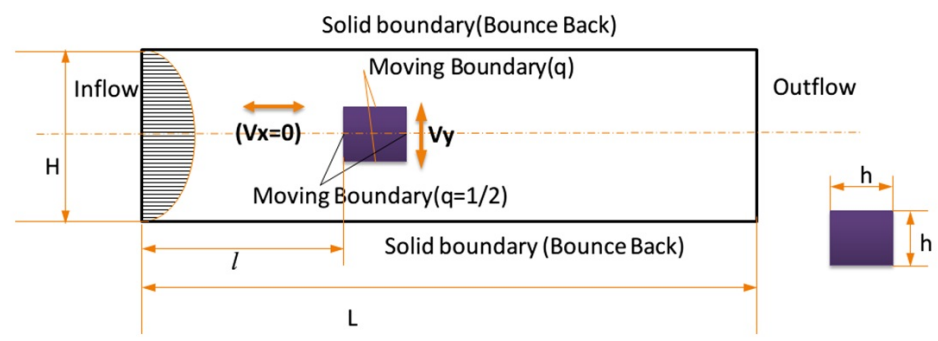

Figure 2: The schematic of the computational domain and its boundary conditions.

[28] A. Ladd, Numerical simulations of particulate suspensions via a discretized boltzmann equation. part 2. numerical results, Journal of Fluid Mechanics 271 (1994) 311-339. doi:10.1017/S0022112094001783 


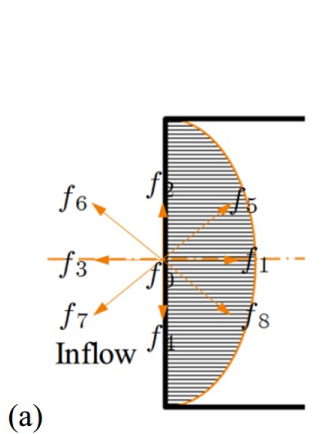

(a)

(b)

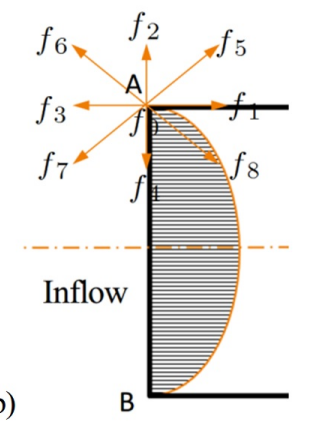

Figure 3: The schematic of left velocity specified boundary(a) and its top corner boundary(b).

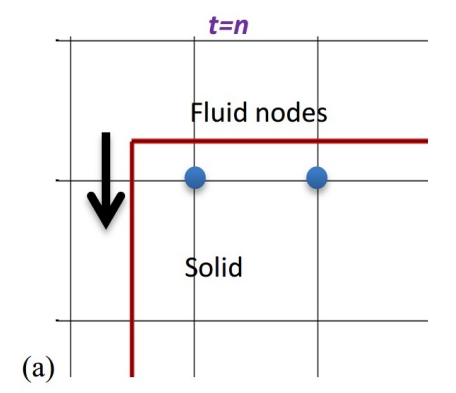

(b)

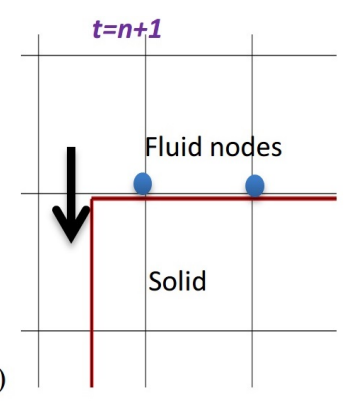

Figure 4: Schematic of the solid nodes translating to the fluid nodes.

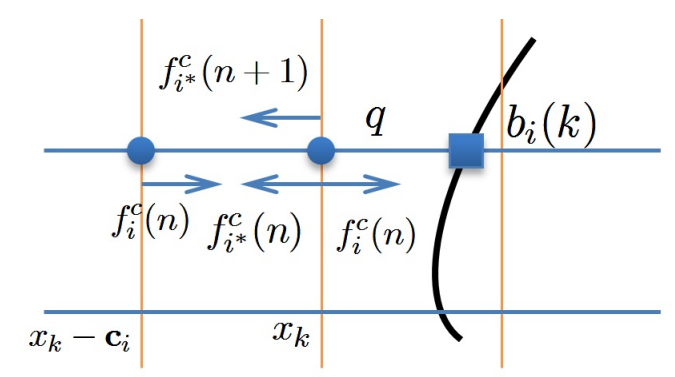

Figure 5: Schematic of the boundary node and its populations in Momentum-Exchange Algorithm(MEA). 


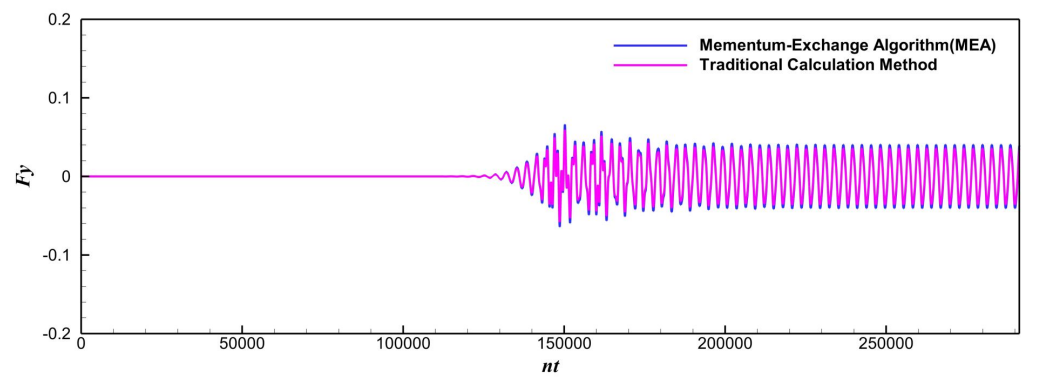

Figure 6: Lift history calculated by the traditional calculation method and Momentum-

Exchange Algorithm $(\operatorname{Re}=100, \beta=1 / 8,1920 \times 320)$. 


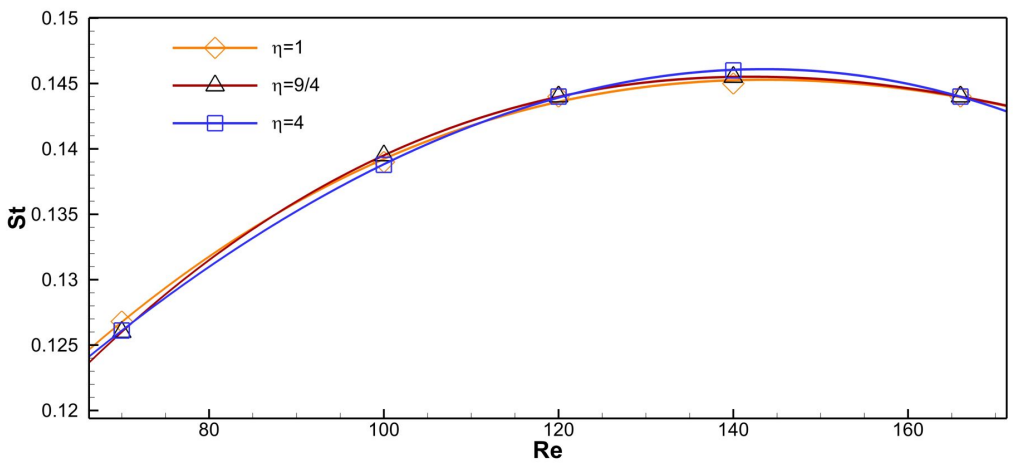

Figure 7: Strouhal number at different Reynold number under different grid number $(\eta=1$ is the case with $960 \times 160)$.

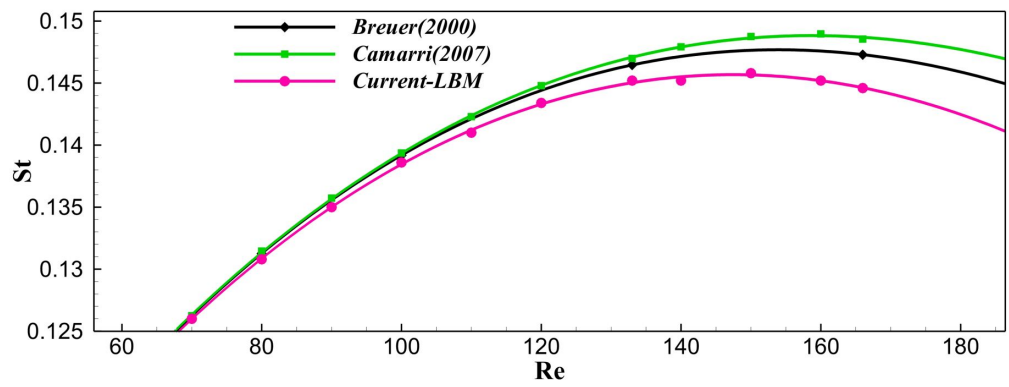

Figure 8: Strouhal number at different Reynold number using current LBM and traditional CFD results (the blockage ratio $\beta=1 / 8,1920 \times 320$ ).

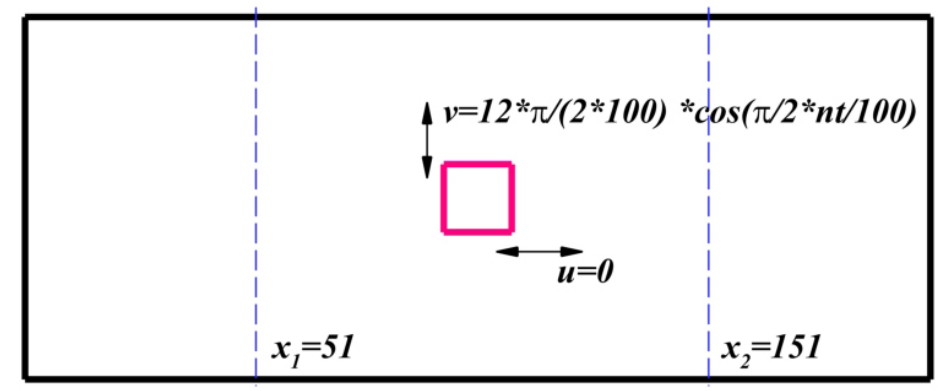

Figure 9: Schematic of the computational domain and initial position of the moving square cylinder(transverse velocity specified) in the quiescent background flow. 


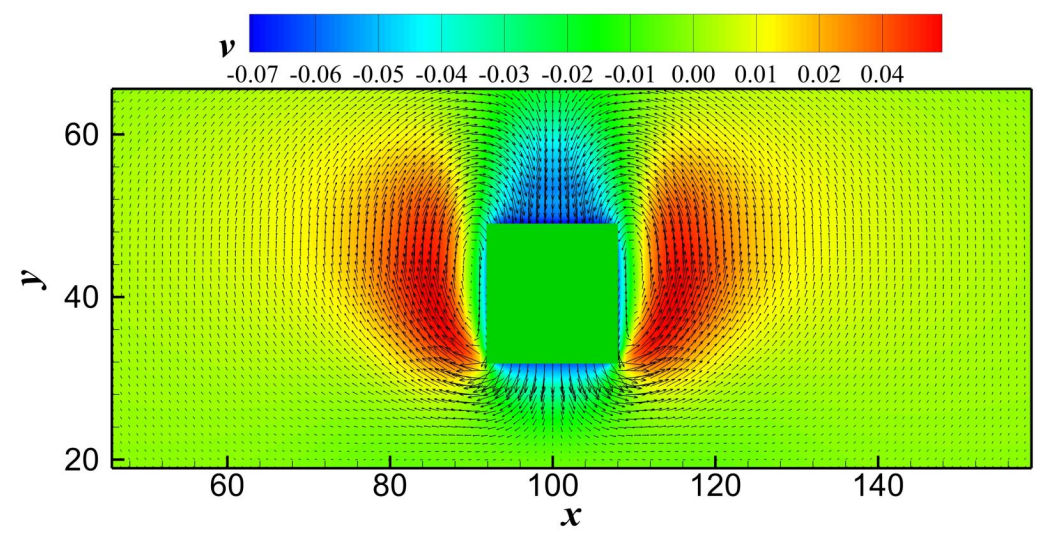

Figure 10: Velocity vector and distributions of v-component of velocity.

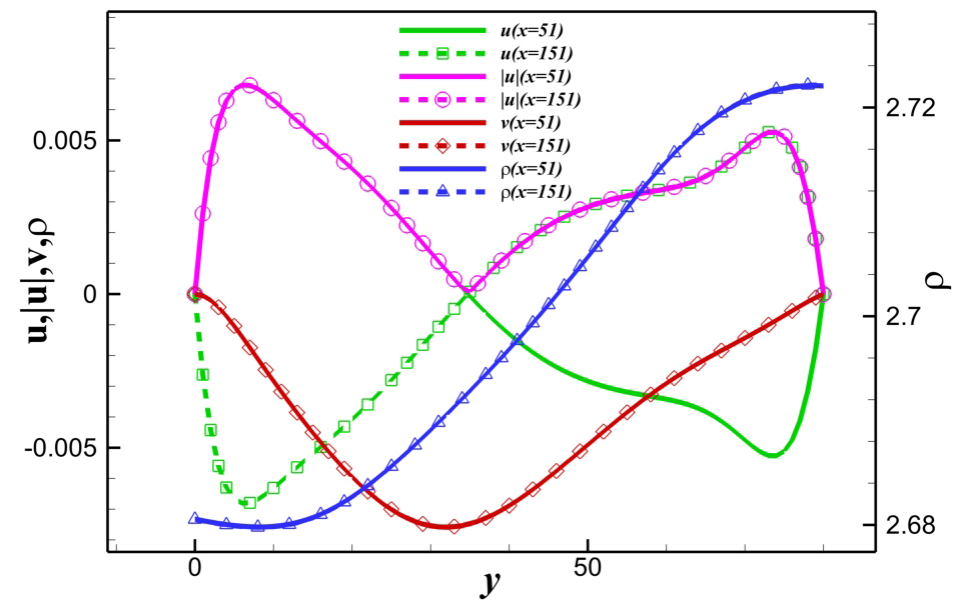

Figure 11: Distributions of the velocity component $u$ and $v,|u|$ and $\rho$ (pressure) at symmetric $\operatorname{points}(x=51,151)$. 


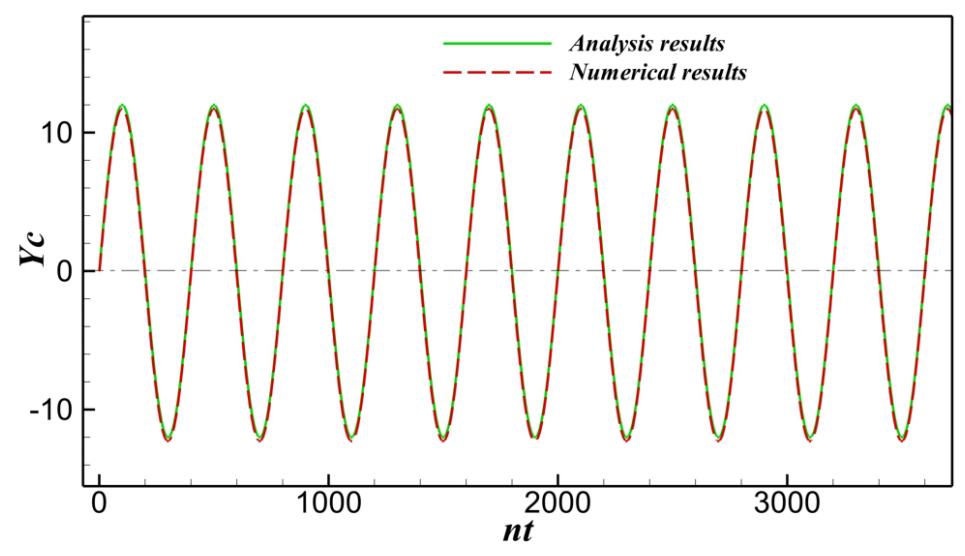

Figure 12: The displacement of the centroid obtained numerically by using LBM and through analysis by using $Y_{c}=12 * \sin \left(\frac{\pi}{2} * \frac{n t}{100}\right)$.

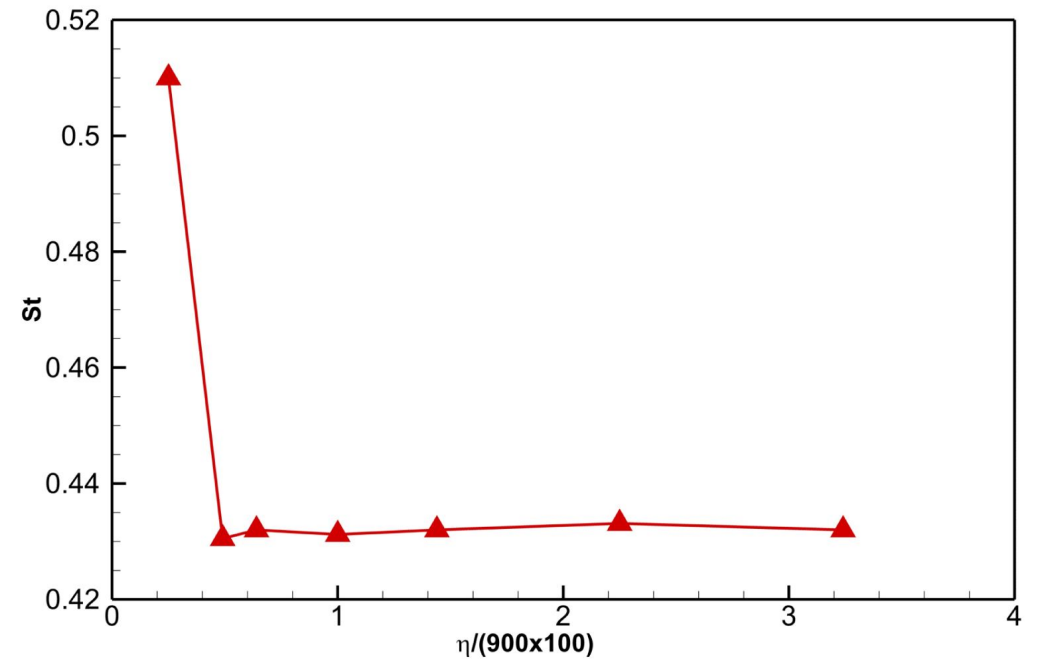

Figure 13: Strouhal numbers at different grid number $(\eta=1$ is the case with $900 \times 100)$. 


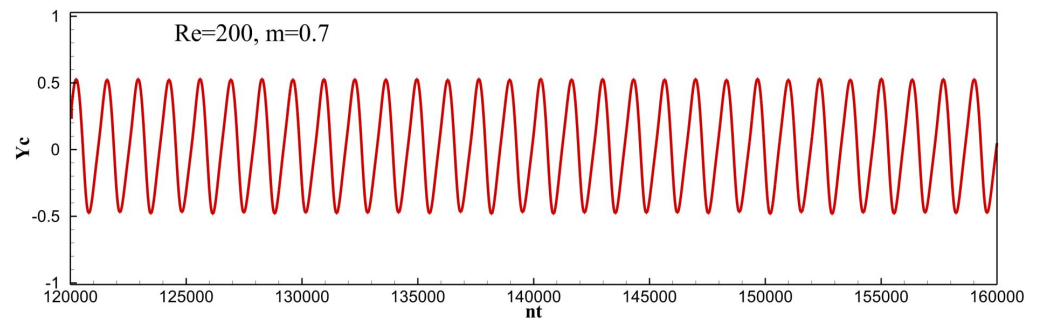

Figure 14: The displacement of the cylinders centroid with time $(\operatorname{Re}=200, m=0.7)$.

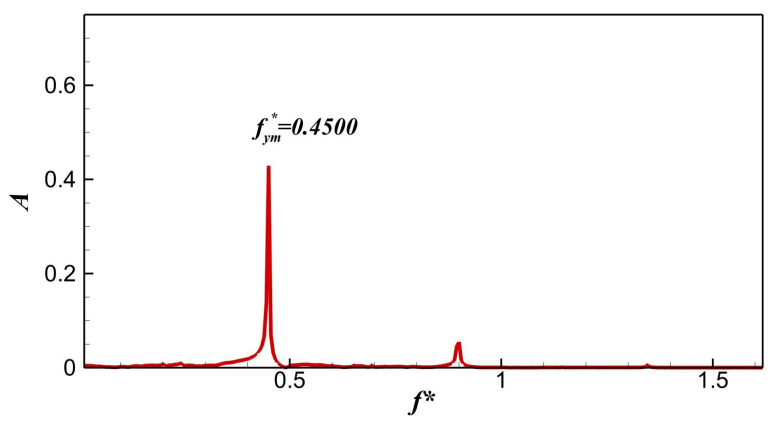

Figure 15: FFT of the cylinder centroids displacement $(\operatorname{Re}=200, m=0.7)$.

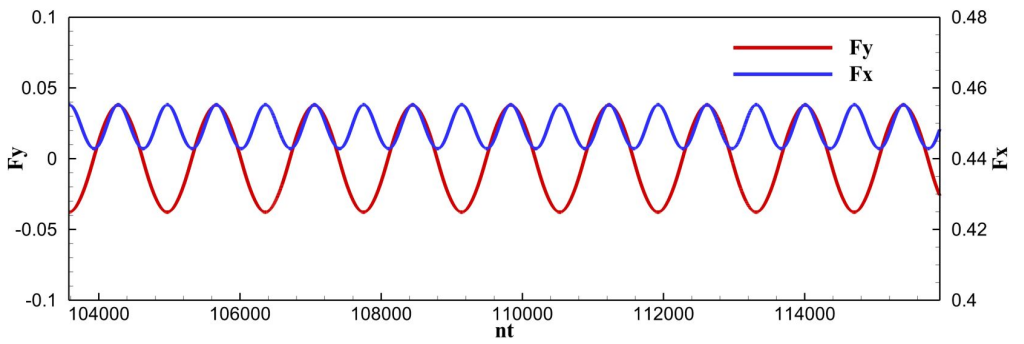

Figure 16: History of the lift force (Fy) and drag force (Fx) of the fixed cylinder. 


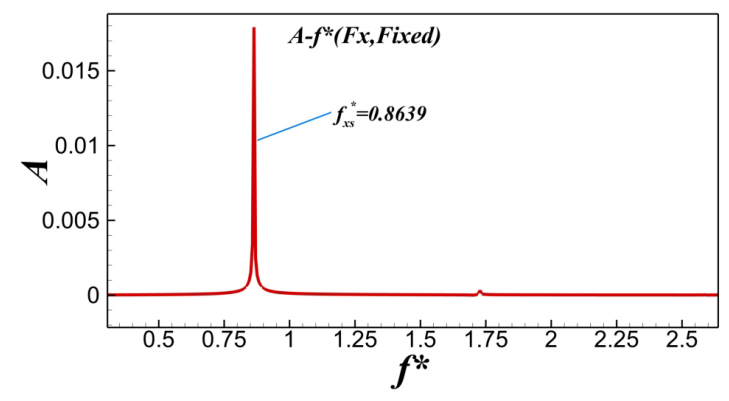

Figure 17: FFT of the drag force (Fx) of the fixed cylinder.

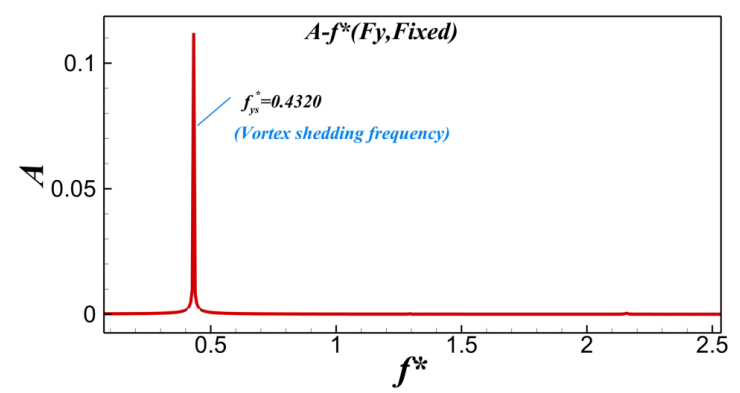

Figure 18: FFT of the lift force (Fy) of the fixed cylinder.

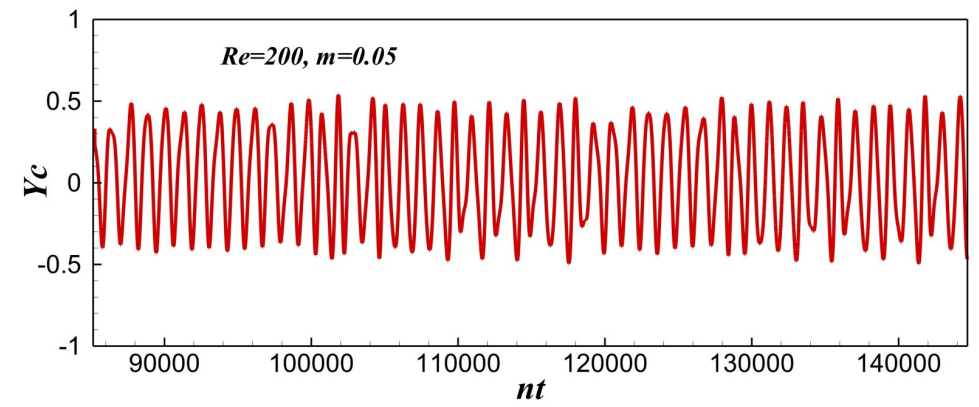

Figure 19: The displacement of the cylinder's centroid with time step $(\operatorname{Re}=200, m=0.05)$. 


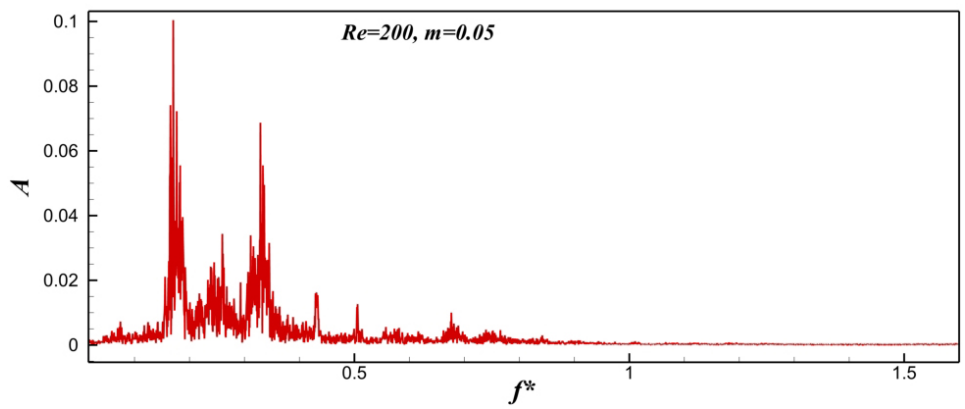

Figure 20: $\quad$ FFT of the cylinder centroids displacement $(\mathrm{Re}=200, m=0.05)$. 